\title{
Intelligent smart home energy efficiency model using artificial TensorFlow engine
}

Hana Jo and Yong Ik Yoon ${ }^{*}$

\author{
*Correspondence: \\ yiyoon@sookmyung.ac.kr \\ Department of IT \\ Engineering, Sookmyung \\ Women's University, \\ Chungpa-Dong 2-Ga, \\ Yongsan-Gu, Seoul 140-742, \\ South Korea
}

\begin{abstract}
Smart home and loT-related technologies are developing rapidly, and various smart devices are being developed to help users enjoy a more comfortable lifestyle. However, the existing smart homes are limited by a scarcity of operating systems to integrate the devices that constitute the smart home environment. This is because these devices use independent loT platforms developed by the brand or company that developed the device, and they produce these devices based on self-service modules. A smart home that lacks an integrated operating system becomes an organizational hassle because the user must then manage each device individually. Furthermore, this leads to problems such as excessive traffic on the smart home network and energy wastage. To overcome these problems, it is necessary to build an integrated management system that connects loT devices to each other. To efficiently manage loT, we propose three intelligent models as loT platform application services for a smart home. The three models are intelligence awareness target as a service (IAT), intelligence energy efficiency as a service (IE $\left.{ }^{2} \mathrm{~S}\right)$, and intelligence service TAS (IST). IAT manages the "things" stage. IAT uses intelligent learning to acquire a situational awareness of the data values generated by things (sensors) to collect data according to the environment. IE ${ }^{2} S$ performs the role of a server (IoT platform) and processes the data collected by IAT. The server uses Mobius, which is an open-source platform that follows international standards, and an artificial TensorFlow engine is used for data learning. IE ${ }^{2} S$ analyzes and learns the users' usage patterns to help provide service automatically. IST helps to provide, control, and manage the service stage. These three intelligent models allow the loT devices in a smart home to mutually cooperate with each other. In addition, these intelligent models can resolve the problems of network congestion and energy wastage by reducing unnecessary network tasks to systematically use energy according to the loT usage patterns in the smart home.
\end{abstract}

Keywords: Smart home, loT platform, Integrated operating system, Intelligent models, Awareness, Energy efficiency, Service, Learning, Automation

\section{Introduction}

Recently, IoT has developed to the extent that it can independently realize and analyze its surrounding environment and apply this knowledge in new environments [1-5]. The speed and accuracy of IoT in receiving and processing commands have improved rapidly because of developments in computer performance, internet networks, network 
processing, efficient algorithms, and so on. IoT is experiencing exponential growth owing to the development of various algorithms [6-9].

Smart homes contain a wide variety of IoT applications. If a conventional home is a combination of simple housing and furniture for providing a residence, a smart home aims at providing services that promote the user's enjoyment and satisfaction. A smart home is an extensive aggregation of IoT applications, which goes beyond the simple goal of being a residence and allows people to manage the rhythms of their lifestyle, pursue enjoyment, and use new services [9-14].

Despite the growth in IoT sector and its technologies, many researchers point out that there are problems with network congestion and electricity usage [15-24]. Modern smart home services lead to indiscriminate energy and network usage, and the technologies for minimizing these problems are inadequate. Recently, many research works are being conducted on alternative energy owing to IoT-related battery problems and rapid increase in electrical energy consumption. However, this kind of novel energy development requires long-term, large-scale research; thus, it is a highly uncertain, future-oriented endeavor. Resolving these problems requires autonomous and efficient network processing, as well as a method that can reduce unnecessary energy usage by operating IoT with precision based on the users' usage patterns $[25,26]$.

To resolve these problems, it is necessary to analyze the IoT users' usage data, and an intelligent manager is required as a platform that comprehensively controls and manages this analyzed usage data. Here, the term intelligent manager does not refer to an IoT platform that simply connects IoT devices, but rather a manager that provides services, which create a customized space for the user in line with the purpose of a smart home, and network technology to minimize energy usage $[27,28]$.

Currently, IoT platforms provide intelligent services; however, most of these services simply process large-scale data and handle mathematical calculations more quickly and accurately than a person. A smart home intelligent manager not only handles groups of devices but also provides user-customized services using algorithms that learn and analyze user data. This enables predicting and preparing for various aspects of various circumstances and environments in a smart home. These predictions and preparations allow the IoT applications to be operated and controlled according to the analyzed data so that network usage and energy wastage can be minimized. Furthermore, the user's feelings, thoughts, and needs are predicted to provide a pleasant and comfortable environment through automated services $[29,30]$.

This study considers other studies to understand the latest technology and trends in various IoT platforms, and to recognize the requirements for providing intelligent IoT energy efficiency services in a smart home. Through this related research, this study is able to propose a service model that possesses the artificial intelligence required to increase network and energy efficiencies.

\section{Related research}

\section{IoT platform}

As IoT has become more diverse, there has been more research on IoT platforms that can integrate IoT devices and make them compatible [31]. An IoT platform is a technology that sets the network standards and data sources of various devices, and integrates 
them regionally and internationally. The goal of an IoT platform is to standardize the devices, to make them compatible with each other, and to integrate them as one. In addition to the international standards organizations, there are various standardization groups and companies that study and develop such technologies.

The international telecommunications union-telecommunication (ITU-T) standardization sector is a major organization in IoT platform research [32]. This organization develops standards for IoT and expects its extraordinary growth in sensor technology, nanotechnology, and embedded intelligence technology. Specifically, one major research effort involves designing a standard structure for service layers and deciding upon an outline for the API and protocols of each service layer. Further, standards for the requirements and management of identifiers in a mobile or internet environment are being developed. In this area, the development of standards related to numbering, naming, and addressing is very important. Currently, standards are being developed for the analysis of interfaces that manage virtual resources in a cloud computing environment. Another important research field is the development of standards related to test structures and requirements for interoperability, including M2M and IP communications [33].

Korea electronics technology institute (KETI) [39] is another major organization in international IoT platform research. KETI unveiled the current IoT platform Mobius 2.0. This organization collaborates with Europe, the United States, and various other countries. Mobius follows the standards of the international standards organization oneM2M, and it supports various applications and services, such as smart cities, smart grids, home automation, and health services. The unique feature of the Mobius IoT platform is that it follows a client-server computing model and is based on the RESTful architecture. The RESTful method is a transmission method derived from the REST method's typical IoT state. It has the benefit of making it easy for the users to uniformly set up and manage various formats and methods of resources.

This kind of IoT platform research follows international standardization documents and supports various technological infrastructures and setups; however, it makes it difficult for the user to intuitively view data, and its usage convenience and management freedom are very inadequate. Further, many international standards organizations and companies use separate standardized rules; thus, there is a very wide variety of standards. This means there are multiple independent IoT technologies, which leads to great difficulties in integrating them into one. In addition, these IoT standards are inadequate in terms of offering services and features that provide an intensive, well-defined model for home network performance and energy efficiency. The existing IoT platforms can be used profitably in various industrial settings and fields; but unlike these, a smart home is a field that connects directly to people, which makes it very intricate and highly unpredictable. Thus, it is necessary to build an IoT platform service model that optimizes smart home networks and energy.

\section{Intelligent loT energy efficiency services in smart homes}

A smart home uses more home appliances than a normal home and consumes energy accordingly. Furthermore, as the scales of apartment buildings become larger, network efficiency must be considered alongside energy efficiency, if there is a system that 
manages the buildings' appliances as one. Thus, various energy and network efficiency services for smart homes are being studied. This research can be considered as divided into two perspectives: physical research and systems research.

On the one hand, there are currently many studies on physical energy efficiency that aim to use clean energy (called green energy) to avoid harming nature and overcome the inadequacies of conventional energy by creating alternative energy [34].

For example, solar energy has often been used to replace electricity and natural gas used in smart homes. By using solar energy, pollutants that harm the environment are not emitted, and the finiteness of conventional gas and electricity can be overcome. In doing so, the energy used by various IoT applications is provided, and more environment-friendly energy consumption becomes possible [15-24].

On the other hand, among the various studies on systematic energy efficiency, there are many studies being conducted on the concept of a smart grid. A smart grid refers to a next-generation intelligent electrical grid. It is a service that allows the electricity supply to be managed effectively by providing electricity providers and producers with information about the electricity users. The users also receive this information; thus, they can manage their individual electricity use and be provided with a high-quality electrical service $[35,36]$.

There are various reasons for the smart grid research to continue. The existing electrical grids are analog and electro-mechanical, and the structure for controlling the grid is limited owing to their centralized systems. Further, if a problem occurs, a large amount of time and manpower are required for repair because of problems related to manual repair systems. A smart grid system is an intelligent and digital approach that enables overcoming these problems. It can meet the consumer's various needs by understanding them through two-way real-time data exchanges. It avoids a centralized system; thus, its distributed network structure is very flexible, and it has a fast and automatic repair system that works through two-way data exchanges. Currently, smart grid research is being performed in various industries.

Smart grid technology can be very beneficial to use in smart homes as well. Smart home systems that use a smart grid can check the electricity consumed by all the devices used by the user, and this data is collected on the server in real time. By collecting detailed data on electricity use, the user's electricity usage patterns can be analyzed. Research is being performed on various systems that enable the use of analysis results to provide electricity efficiently $[35,36]$.

Building a smart grid requires energy storage systems (ESS), advanced metering infrastructure (AMI), energy management systems, electric car and charging stations, distributed power supply, new-renewable energy, two-way data communication technology, intelligent power transmission-supply systems, and so on. Among these, the ESS is the most essential part of an IoT network. Previously, existing leftover electrical energy was simply wasted. However, ESS helps store large amounts of electricity. This can be used to store energy, adjust consumption, and supply on requirements to reduce energy wastage. The AMI is actively being studied as a method to adjust consumption and supply as needed. The AMI sets electricity usage amounts and discerns each user's usage patterns based on the usage data from each household. This data can be used to provide optimal electricity, and it can lower electricity costs and prevent wastage. The data is transmitted 
to an AMI data collector via each household's Wi-Fi network, and it is gathered together by TropOS mesh radio, which is broadcast throughout each region. However, analyzing energy usage patterns and collecting electricity usage data is a very simple form of data collection and analysis.

Various energy efficiency models that use intelligent learning are being studied. However, these systems still analyze only user patterns and gather electricity using data en masse. This is merely statistical and numerical analysis of the gathered data. Understanding the patterns of each user requires a system that meaningfully processes and analyzes the user's individual data rather than mass data. It must provide customized service for each user or each device in each household by individually analyzing a variety of collected user data and learning from data which is collected in real time. This is a method for saving and efficiently using energy, and it increases user satisfaction. In the future, more research is needed to study specialized artificial intelligence learning models that are used as smart home energy services which can be individually customized.

\section{Definitions of research concepts}

\section{Intelligence awareness target as a service concept}

Intelligence awareness target as a service (IAT) is a model that performs intelligent situational awareness data processing at the level of things [31]. IAT collects data generated from things, gathering only the data that is needed according to pre-determined situations. This avoids unnecessary processing and performs the required actions and processes so that it is possible to process data in a way that is appropriate for the situation.

IAT defines four major types of user activities and lifestyle patterns so that it can preemptively become aware of situations. Based on these, IAT becomes aware of the situation and processes the required data.

This study divides IAT into sensor IAT, smart phone IAT, and smart appliance IAT. These three devices (sensor, smart phone, and smart appliance) are very easy to access and collect smart home environmental data and user data, and they are some of the devices that perform the most data generation and processing. In large, the IAT can be divided into the stationary IAT and the non-stationary IAT. The stationary IAT typically deals with a sensor IAT and non-stationary IAT is frequently related to a smart phone IAT.

Meanwhile, a smart appliance IAT can also be explained as another type of IAT. It is distinguished from other types of IAT in that it is not only a form of IAT but also a type of IST (Intelligence service target as a service). Since the smart appliance IAT is different from other IATs in this regard, this paper discusses it as an additional type of IAT.

\section{Sensor IAT}

Sensor IAT is a stationary things model with the purpose of collecting simple sensor data. It is a group of sensors that are required to determine the situation indoors and present definite numerical values and concepts about the environment.

Figure 1 is the floor plan of a smart home that has stationary sensor boards installed in each space. The minimum number of sensors required to determine the current status of the smart home is attached to the smart boards, and they collect actual data. Three kinds of sensors are used: sound, motion, and rotary. The purpose of these sensors is to gather 


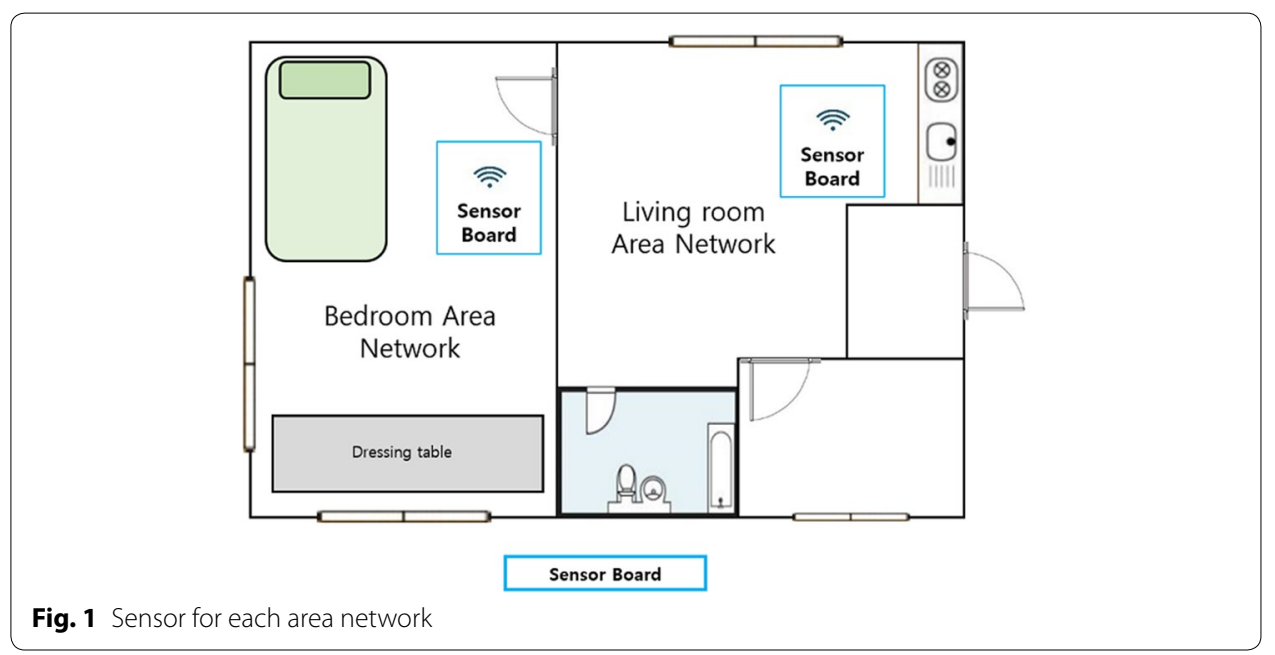

\begin{tabular}{|c|c|c|c|c|}
\hline $\begin{array}{c}\text { sensor } \\
\text { board }\end{array}$ & situation $\boldsymbol{a}$ & situation $\boldsymbol{b}$ & situation $\boldsymbol{c}$ & situation $\boldsymbol{d}$ \\
\hline Motion & 0 & $\mathrm{X}$ & $\mathrm{X}$ & $\mathrm{X}$ \\
\hline Sound & 0 & 0 & $\mathrm{X}$ & $\mathrm{X}$ \\
\hline Rotary & 0 & 0 & $\mathrm{X}$ & No user indoor \\
\hline \multicolumn{6}{|c|}{ Result } & $\begin{array}{c}\text { user has indoor } \\
\text { (activating) }\end{array}$ & $\begin{array}{c}\text { user has indoor } \\
\text { (resting) }\end{array}$ & $\begin{array}{c}\text { user has indoor } \\
\text { (sleeping) }\end{array}$ \\
Fig. 2 Type of situation according to sensor
\end{tabular}

data and become aware of the indoor situation of each of the separate area networks in the smart home individually.

As shown in Fig. 2, user activities and lifestyle patterns are defined into four major situations for the ITA to preemptively become aware of the indoor situation. The first situation is a user performing an activity indoors. The second situation is a user resting indoors. The third situation is a user sleeping indoors. The fourth situation is that there is no user indoors. The user's location, movements, and patterns can be understood through these categorized situations.

The sensor check shown in Fig. 2 is a very simple action, which determines if something has been detected or not, and it does not require a large amount of data transmission. Thus, IAT can quickly determine the situation beforehand and transmit data to the server only when it is needed. When data is not transferred, the network is not used, and the energy use is minimized.

\section{Smart phone IAT}

Smart phones are the non-stationary IAT as opposed to sensor IAT.

As shown in Fig. 3, smart phones contain various sensors. It is the most important device for collecting data directly from the position closest to the user. Thus, it collects 

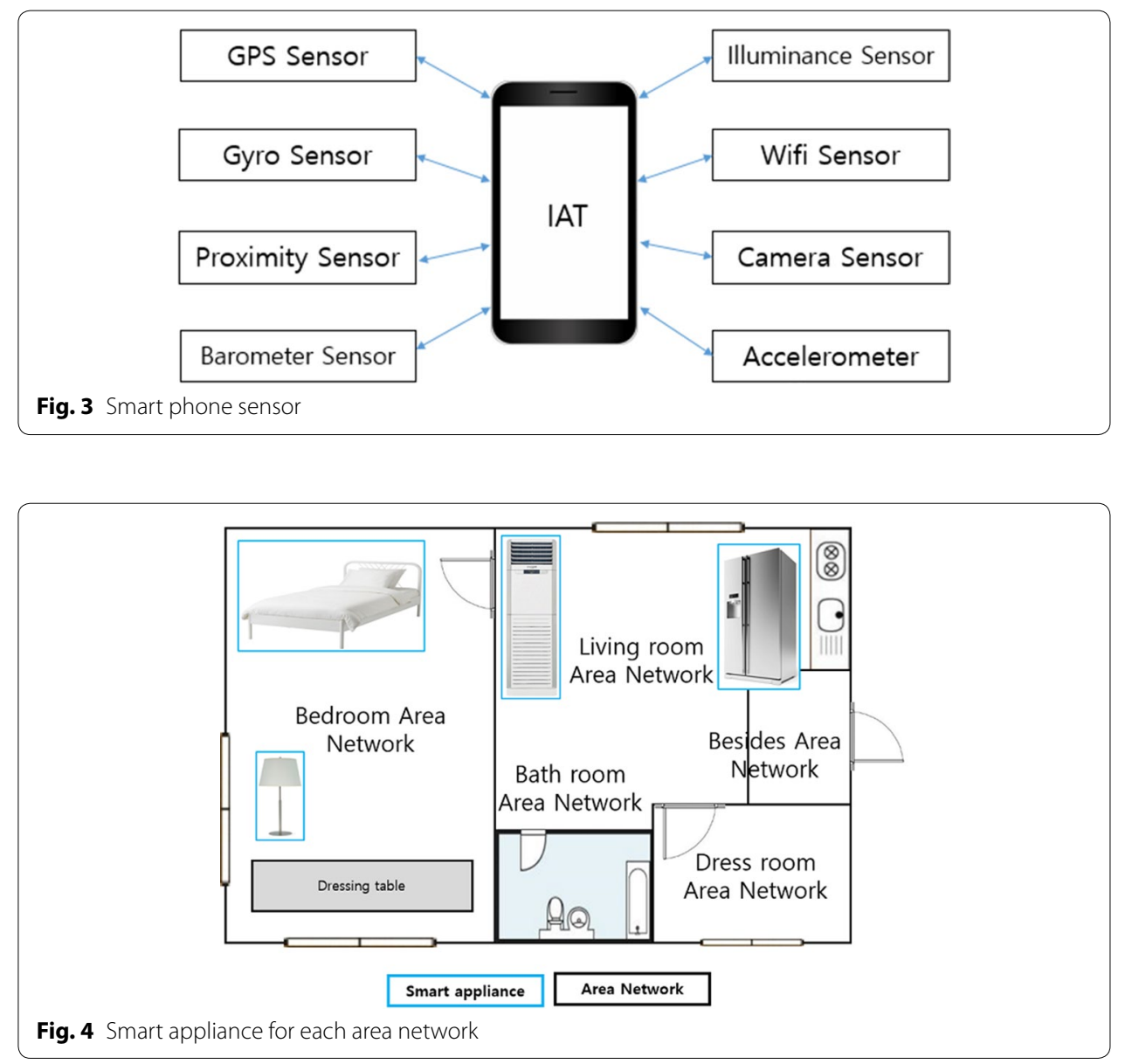

data that is needed to analyze the user's status. This study used the smart phone's GPS sensor, gyro sensor, proximity sensor, barometer sensor, and illuminance sensor. These sensors allow a detailed understanding of the user's position, movement, illumination at the current position, and so on. This data is transmitted to the server and classified by weights as part of the learning data.

\section{Smart appliance IAT}

Smart appliance IAT is a more complex model than the existing things level sensor IAT and smart phone IAT. This can handle both things level IAT as well as service level IST.

Smart appliance IAT has two roles: collecting data from smart appliances at the level of things and providing services at the level of service.

The first role of collecting data can be divided into two parts: collecting environment data with simple sensors and collecting data to analyze users' usage patterns. For the first part, simple environment data is collected according to the functions of the various smart appliances, as shown in Fig. 4; and the collected data comes in various forms. Smart appliance IAT processes the various data to integrate it and transmits it to the server. An appliance, such as an air conditioner, measures the indoor temperature and transmits it to the server. The other part is the collection of user data, and for this part 


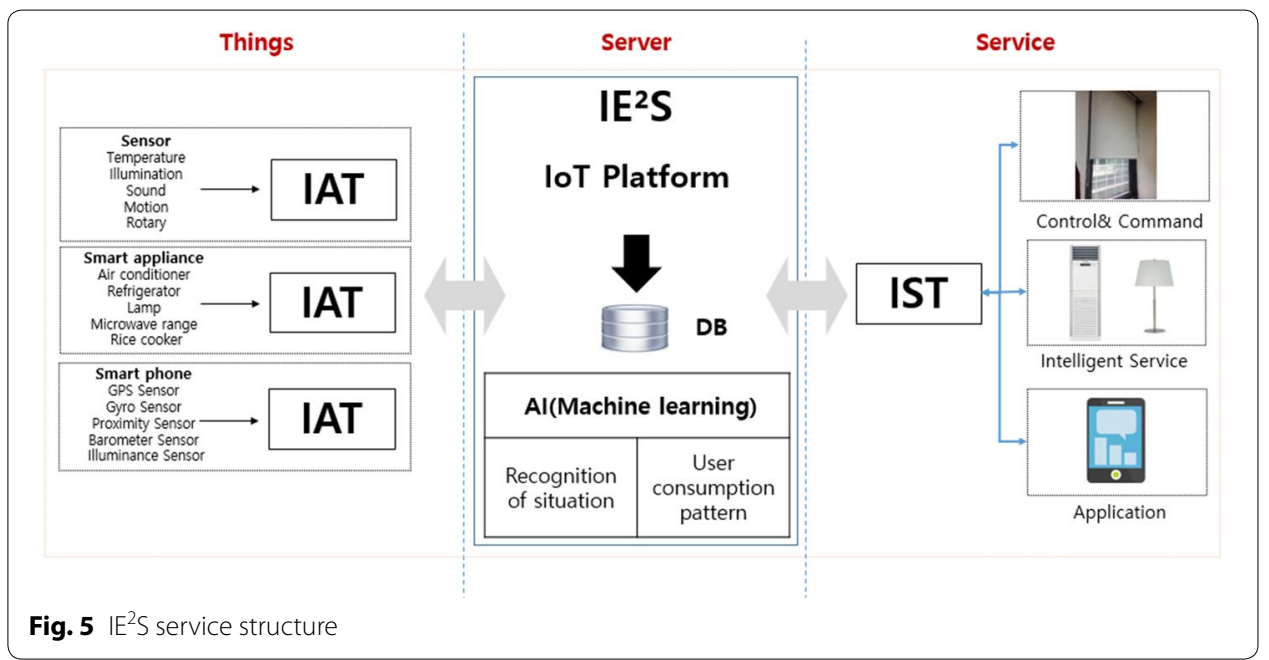

the smart appliance usage activity records are gathered to analyze usage patterns. The collected data is transmitted to the server, and the user patterns are analyzed through machine learning. This is necessary to determine and provide the services that are suitable for these patterns.

The second role is providing automated service as directed by the server. For example, for an air conditioner, the result of a decision on the server could lead to air conditioning service being provided to optimize the indoor temperature.

Smart appliance IAT performs both the data collection and the automated service together. It is a next-generation smart home things model that is capable of two-way communication, and it combines an IAT model that collects data and an IST model that provides services, which are presented in this study. In addition, it also performs a role of IST model since it deals with service level that providing services IST model is presented in the latter part in this paper.

\section{Intelligence energy efficiency as a service concept}

Intelligence energy efficiency as a service $\left(\mathrm{IE}^{2} \mathrm{~S}\right)$ is the IoT platform and performs the role of a server. In Fig. 5, $\mathrm{IE}^{2} \mathrm{~S}$ intelligently integrates and controls both the things level and the service level.

On the things level, IAT becomes aware of situations beforehand and transmits collected data to $\mathrm{IE}^{2} \mathrm{~S}$. The transmitted data is continuously collected and a neural network learning algorithm is used to analyze it. $\mathrm{IE}^{2} \mathrm{~S}$ performs two functions through machine learning: recognizing of situation and finding out user consumption pattern [37, 38].

For the first function, $\mathrm{IE}^{2} \mathrm{~S}$ continuously collects the data sent from IAT in real time and analyzes the situation within the smart home to recognize situation. It not only becomes aware of the situation but also analyzes several kinds of environmental data, and it finds the optimal situation conditions. For the second function, it examines the user's IoT usage patterns within the smart home. Data from the user operating the IoT or previously recorded data is collected and used as target data for learning. Learning is performed continuously to provide the situations within the smart home environment that the user wants. 


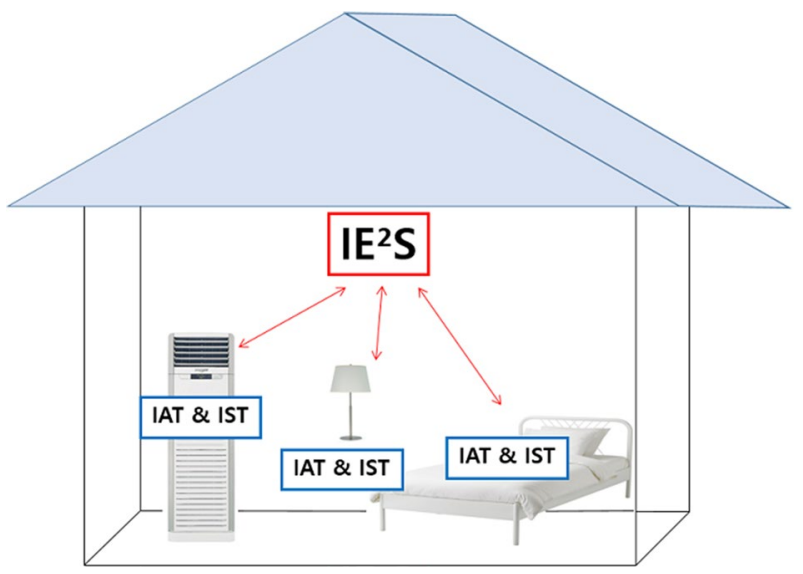

Fig. 6 IST and IE 2 S communications for smart appliances

On the service level, $\mathrm{IE}^{2} \mathrm{~S}$ analyzes the learned data and provides services which are suitable to the corresponding situations. To do this, service command data is transferred from $\mathrm{IE}^{2} \mathrm{~S}$ to IST. Actions are not simply performed, but rather actions are defined by the IST system to provide services which are suitable to device features, and actions are performed in accordance with these features. In analyzing the user's usage patterns within the smart home and avoiding unnecessary tasks, the goal is to reduce energy and network usage. If unnecessary services are reduced and necessary services are provided, smart home energy efficiency automation can be achieved.

\section{Intelligence service target as a service concept}

Intelligence service TAS (IST) has the role of providing services through various smart appliances. IST uses the learning data from $\mathrm{IE}^{2} \mathrm{~S}$ to provide the required services to the user. Since, data collection and service provision are usually performed by one device, so both IAT and IST are included in the smart appliances, as shown in Fig. 6.

In Fig. 6, there are three devices in the IST: the air conditioner, lamp, and electric blanket. To show a large amount of data and various services involved, the smart devices that are most often used in a household were selected from the smart appliance category. The air conditioner transmits the current indoor temperature to the server and the user's usage patterns and data are learned simultaneously in $\mathrm{IE}^{2} \mathrm{~S}$ to select a suitable temperature level. As a result, an optimal indoor temperature can be maintained through air conditioning. The lamp adjusts its intensity and operating status according to the current time and the presence or absence of a user indoors, and the user's usage patterns are analyzed to maintain the optimal values. The electric blanket checks the user's body temperature and the indoor temperature, and it continuously collects and analyzes the increase and decrease adjustments made by the user to provide the optimal temperature.

In addition to these intelligent services, IST can also control or operate simple device actions, and it can provide various application services to the user through smart phone applications. However, this study is specifically focused on intelligent services only. 


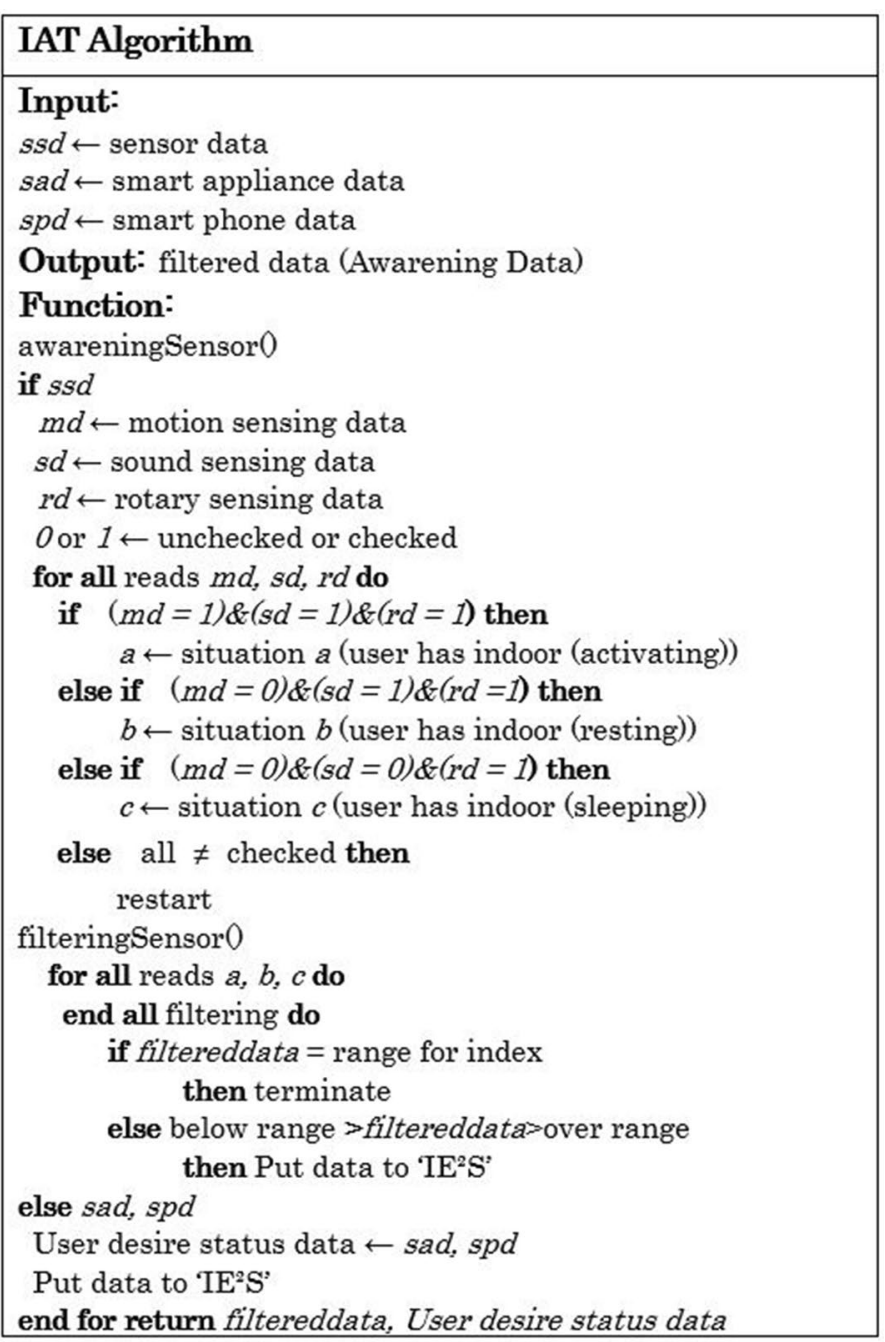

Fig. 7 IAT algorithm

\section{Intelligent model algorithms}

\section{IAT algorithm}

The IAT algorithm is designed to transmit data from the things level to the server with a minimal activity. To perform minimal activity, it becomes aware of the situation beforehand and gathers the necessary data. The necessary data is gathered and transmitted to the server. In this case, it can reduce the data collection activities for unnecessary things, and only important data is processed on the server.

In Fig. 7, three types of data (data from sensor, smart phone, smart appliance which or explained with IAT concept) are entered as input in the IAT algorithm. There are two cases: when the sensor data is entered and when the smart appliance or smart phone data are entered. In this section, data gathered from a smart appliance or a smart phone are set as a desire status data because those data are helpful in finding out what users want by letting users control the device. 


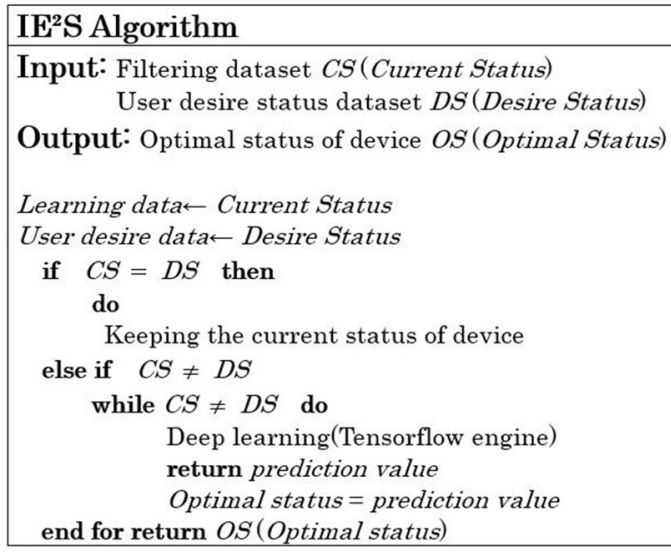

First, when the sensor data is entered, the current situation in the smart home is classified into four types. These four types are also shown in Fig. 2. This situation is classified so that the sensors and things only operate when there is a user present indoors. The activated sensor filters the data that is outside the standard range and transmits the rest to the server.

Second, when smart appliance data or smart phone data are entered, this data is considered data that the user desires, and it is transmitted to the server. The values of this data are adjusted as the user uses the smart appliance and changes them to the desired values according to their needs. By doing so, it can assume what kind of situation the user wants to change and what kind of situation the user desires.

\section{$\mathrm{IE}^{2} \mathrm{~S}$ algorithm}

As a server, $\mathrm{IE}^{2} \mathrm{~S}$ gathers the data sent from IAT, processes it, and performs learning, as shown in Fig. 8. It receives the filtered and user-desired data sent by IAT as input. To learn from the data, it sends the current status to Learning data and the desired status to user desire data, as shown in the image. If there is a difference between the current status and the desired status, $\mathrm{IE}^{2} \mathrm{~S}$ predicts the optimal value through deep learning. To perform learning, an artificial TensorFlow engine is used to increase the accuracy of the prediction value. This allows $\mathrm{IE}^{2} \mathrm{~S}$ to create an optimal status through predictions, even if the User desire data input was ultimately not received when the input data arrived.

\section{IST algorithm}

In Fig. 9, IST maintains or changes the current status of devices through commands received from $\mathrm{IE}^{2} \mathrm{~S}$. IST is an algorithm that quickly performs the orders transmitted from $I^{2} S$. IST performs the role of creating a mutual connection between $\mathrm{IE}^{2} \mathrm{~S}$ and devices, and it is continuously preparing to receive commands. Finally, it transmits the changed device status back to $\mathrm{IE}^{2} \mathrm{~S}$ and continuously checks the data on the current status. 


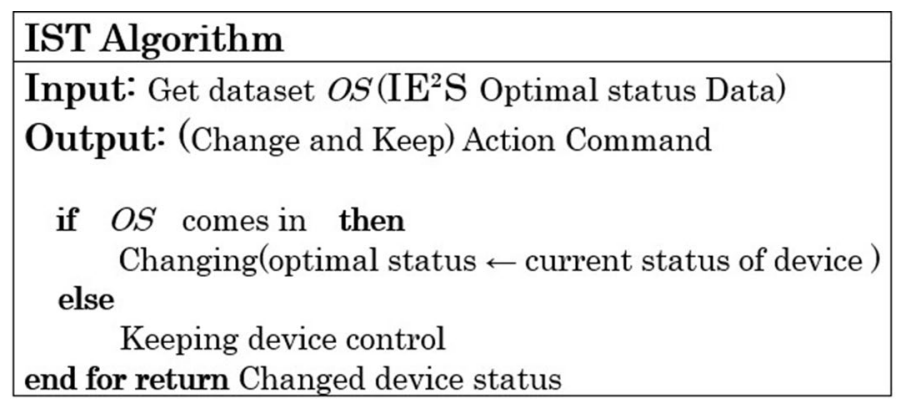

Fig. 9 IST algorithm

\begin{tabular}{|ccc|}
\hline Sensor / Actuator & $\begin{array}{c}\text { Port on Grove Base Shield } \\
\text { Used }\end{array}$ & Function0 \\
DF ROBOT-Sound Sensor & A0 & int readSound); \\
DF ROBOT-Motion Sensor & A1 & int readMotion0; \\
DF ROBOT-Rotary Sensor & A2 & int readRotary); \\
Fig. 10 IAT/IST algorithms build for Arduino boards & & \\
\hline
\end{tabular}

\section{Implementation and analysis}

\section{Things (sensor)}

The sensor board in the smart home uses an Arduino board [31] and the three sensors shown in Fig. 10. IAT is modularized as a single library and includes all the necessary sensor action codes. This is useful for managing the various sensors in an integrated manner. The Arduino board reads the data through serial communication with the sensors. The MQTT protocol is used for Wi-Fi communication between the Arduino board and the server. It is aware of the current IoT situation in real time and transmits the required data to the server.

Figure 11 shows the Arduino code that uses the IAT algorithm. The sensor attached to the sensor board detects the environment. The Awareningloop function creates condition statements (if-else), which can continuously monitor the situation. It is in an event format so that even though it is a loop, it does not incur a large processing cost when events do not occur. Through the filtering function, data that is outside the standard range is sent to the server when situation conditions are established, so that only the required data is sent even if the algorithm is aware of a situation.

\section{Server}

The server uses the $\mathrm{IE}^{2} \mathrm{~S}$ algorithm and the Mobius [39] international standard platform. On the Mobius server, the data received from IAT is converted into a database through MySQL. This data is learned by the TensorFlow engine [40] using the Jupyter editor [41] with the Anaconda package.

A system was built as a simple example of implementation, which collects indoor temperature data and compares it to the user's preferred temperature values to learn the user's desired optimal indoor temperature and provide service. 


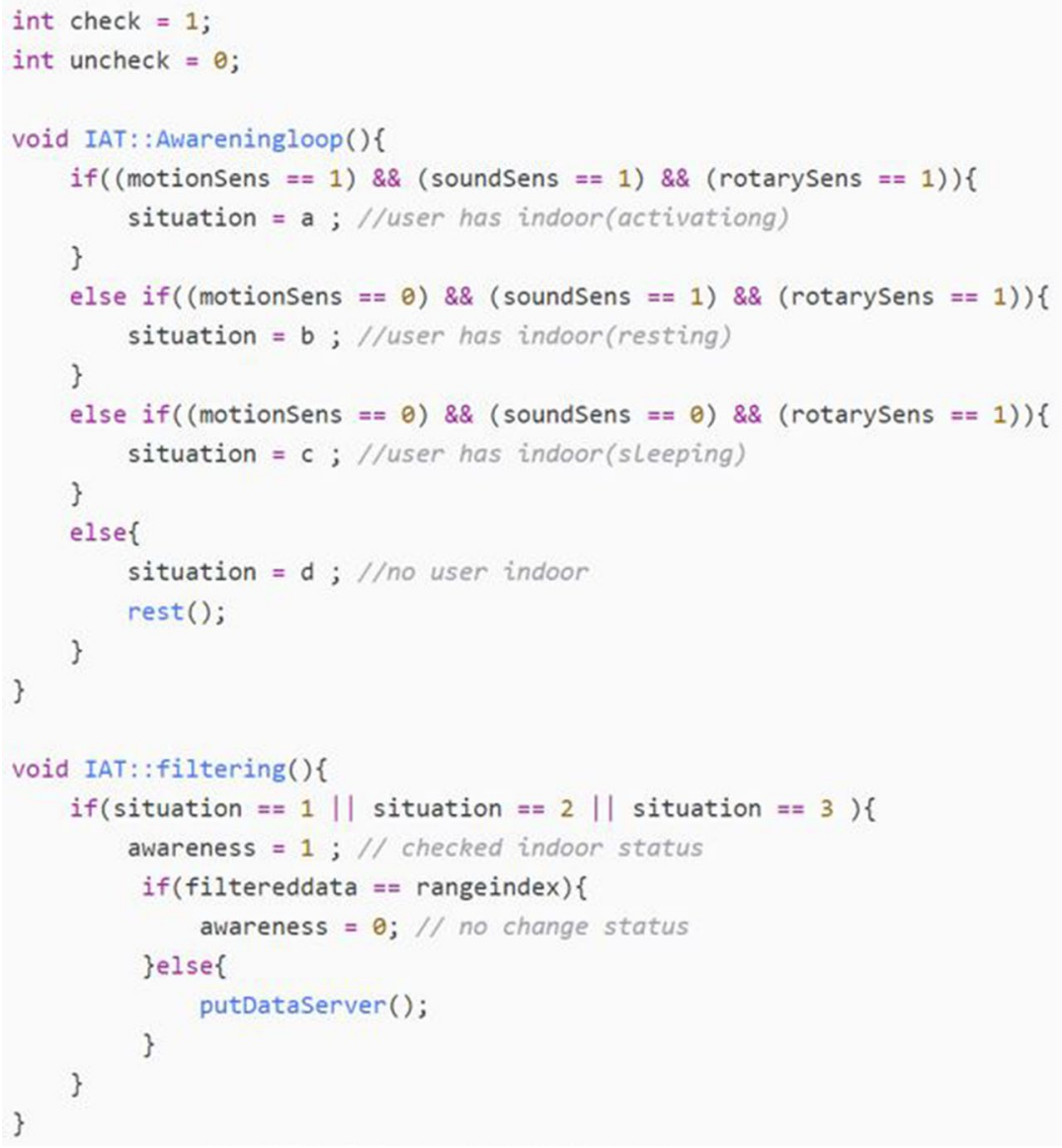

Fig. 11 Arduino code using IAT functions

\begin{tabular}{|c|c|c|}
\hline Month & Average temp $\left({ }^{\circ} \mathrm{C}\right)$ & User desire temp \\
\hline 1 & -1.8 & 25 \\
\hline 2 & -0.2 & 23 \\
\hline 3 & 6.3 & 22 \\
\hline 4 & 13.9 & 22 \\
\hline 5 & 19.5 & 23 \\
\hline 6 & 23.3 & 22 \\
\hline 7 & 26.9 & 21 \\
\hline 8 & 25.9 & 20 \\
\hline 9 & 22.1 & 22 \\
\hline 10 & 16.4 & 23 \\
\hline 11 & 5.6 & 25 \\
\hline 12 & -1.9 & 26 \\
\hline
\end{tabular}

Fig. 12 Monthly average temperature in Seoul and user preference temperature

Figure 12 shows the weather information provided by the Seoul city weather service for 2017. It shows the standard values for the current status of the environment in terms of monthly average temperatures, and these are the standard temperature values used 


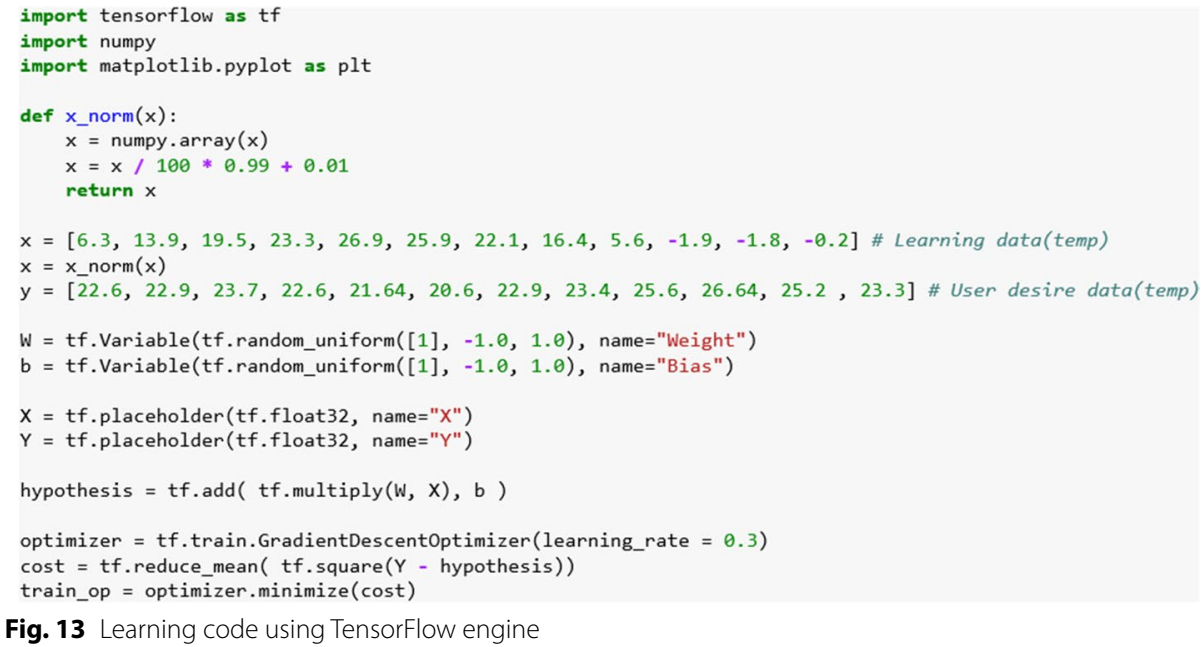

for learning. The "user desire temp" values are data collected by the smart appliance air conditioner. The temperature differences at which the user turned on the air conditioner were recorded, and the user's preferred temperature patterns were hypothesized. This information is separately digitized and processed to create a dataset suitable for learning. The data is learned by the TensorFlow engine and the final indoor temperature is found.

Figure 13 shows the learning code that uses the TensorFlow engine. The monthly average temperatures are entered as values of $\mathrm{x}$ as learning data, and the user-preferred temperature data is entered for $\mathrm{y}$. In the process of turning $\mathrm{X}$ into $\mathrm{Y}$, the relationships are continuously estimated, and operators suitable for these relationships are found. When a new $\mathrm{X}$ value is entered, a final output value is created through the learned operators. The test was performed with a learning rate of 0.3 and 1000 rounds of learning.

Figure 14 shows the process of performing learning 1000 times. It can be seen that the cost starts as 513 and is gradually reduced until it reaches 1.06.

Figure 15 shows the results of performing the test using the learning program. First, the monthly temperature data is randomly entered as different values of $x$. The prediction values can be confirmed by the learning algorithm. The output values are very similar to the user-preferred temperatures.

Figure 16 shows a graph of the final results. The blue line is the monthly average temperature and the black line is the user-preferred temperature. The red line shows the final values estimated by learning the relationship between these two pieces of data. According to this graph, the black and red lines are very similar when both are compared to the blue line. If more data is used for learning, the error will be reduced further.

\section{Conclusion}

Smart home and IoT-related technologies are developing rapidly, and many smart devices are being developed to help users enjoy a more comfortable lifestyle. However, the existing smart homes are limited by a scarcity of operating systems to integrate the devices that constitute the smart home environment. This is because these devices use 


\begin{tabular}{|c|c|c|c|c|c|c|}
\hline Step: & $\theta$ & Cost: & 513.8344 & W: & [0.99421155] & [14.490607] \\
\hline tep: & 1 & Cost: & 80.231926 & W: & {$[1.6422824]$} & [19.767506] \\
\hline tep: & 2 & Cost: & 14.820018 & W: & [1.8395431] & [21.824331] \\
\hline Step: & 3 & Cost: & 4.9327855 & W: & [1.8620838] & [22.630646] \\
\hline tep: & 4 & Cost: & 3.4191496 & W: & [1.8171172] & [22.951296] \\
\hline tep: & 5 & Cost: & 3.1685658 & W: & {$[1.7462755]$} & [23.083298] \\
\hline Step: & 6 & Cost: & 3.1087363 & W: & {$[1.665724]$} & {$[23.141994]$} \\
\hline 5tep: & 7 & Cost: & 3.0779524 & W: & [1.5817379] & [23.172176] \\
\hline Step: & 8 & Cost: & 3.0518227 & W: & {$[1.4967519]$} & {$[23.191238]$} \\
\hline tep: & 9 & Cost: & 3.0266664 & W: & [1.4117094] & [23.205936] \\
\hline tep: & 10 & Cost: & 3.0019257 & $\begin{array}{l}\text { W: } \\
\text { • } \\
\text { ? }\end{array}$ & [1.3269747] & [23.218893] \\
\hline & 990 & & .007423 & W: & $-11.958592]$ & [25.081951] \\
\hline o: & 991 & Cost: & 1.0694257 & W: & {$[-11.958758]$} & [25.081974] \\
\hline tep: & 992 & Cost: & 1.0694257 & W: & {$[-11.958923]$} & [25.081997] \\
\hline tep: & 993 & Cost: & 1.0694255 & W: & {$[-11.959087]$} & {$[25.08202]$} \\
\hline tep: & 994 & Cost: & 1.0694256 & W: & {$[-11.95925]$} & [25.082043] \\
\hline Step: & 995 & Cost: & 1.0694249 & W: & {$[-11.959413]$} & {$[25.082066]$} \\
\hline Step: & 996 & Cost: & 1.0694246 & W: & {$[-11.959574]$} & [25.082088] \\
\hline Step & 997 & Cost: & 1.0694252 & W: & {$[-11.959734]$} & {$[25.082111]$} \\
\hline Step & 998 & Cost: & 1.0694246 & W: & {$[-11.959893]$} & {$[25.082134]$} \\
\hline Step: & 999 & Cost: & 1.0694246 & W: & {$[-11.960052]$} & {$[25.082155]$} \\
\hline
\end{tabular}

Fig. 14 The process of learning 1000 times

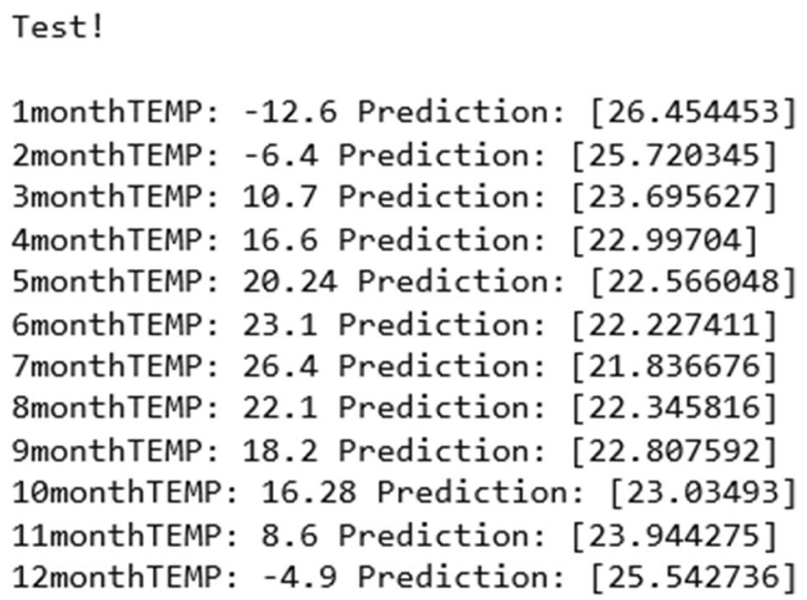

Fig. 15 The result of executing TEST on learned program

independent IoT platforms developed by the brand or company that developed the device, and they produce these devices based on self-service modules. A smart home that lacks an integrated operating system becomes an organizational hassle because the user must then manage each device individually.

The rapid and large-scale development of smart home and IoT-related technologies have caused many problems, such as inefficient operating systems, excessive network traffic, and energy wastage. To overcome these problems, it is necessary to build an integrated management system which connects IoT devices to each other. To efficiently manage IoT, we have proposed three intelligent models as IoT platform application 


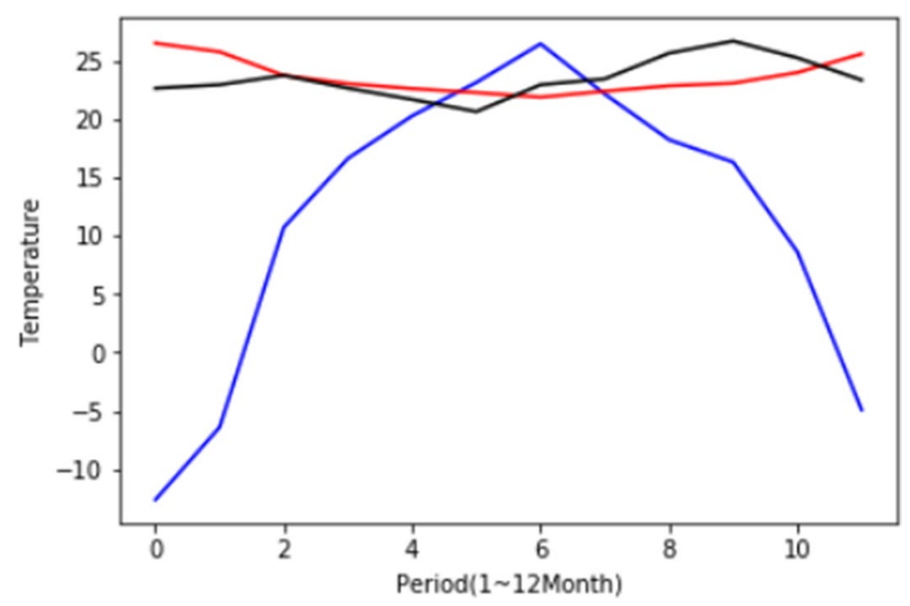

Fig. 16 A graph showing final result

services for a smart home. The three models are IAT, IE ${ }^{2} \mathrm{~S}$, and IST. Through these three models, intelligent learning is performed on the data generated by IoT to make the IoT device aware of current surrounding situations. IAT gathers necessary information according to the known situation and activates IoT. At this point, IoT is activated only when it is needed according to the situation. $\mathrm{IE}^{2} \mathrm{~S}$ performs the role of the server (IoT platform) and uses a TensorFlow engine to learn the data collected by IAT. It learns and analyzes the user's usage patterns to provide optimal prediction data. In the experiment performed in this study, data for monthly average temperature was compared with that for the user's desired temperature and very similar prediction temperature results were produced through deep learning. Even when random values were entered as data for monthly average temperature, it produced prediction values that were very similar to the data of the user's desired temperature. The prediction accuracy increases proportionally as the amount of user data increases, and further research is needed on increasing the prediction accuracy by continuing to collect data in the future. For future research work, the intelligent algorithm's concrete physical structure and features must be studied further, actual data must be collected, and prediction accuracy must be improved for the sake of system stability. Further, direct analysis and testing of the network must be performed. Studying these three intelligent models will allow IoT devices in a smart home to mutually cooperate with each other. This study presented smart home energy efficiency automation services. With more in-depth research on intelligent models in the future, structured intelligent IoT platforms for smart homes will provide not only houses made to be simple indoor residences, but smart, energy-efficient living spaces for healthy lives.

Authors' contributions

$\mathrm{HJ}$ carried out the molecular genetic studies, participated in the sequence alignment and drafted the manuscript. YIY helped in developing a valid analysis of the experts' review and participated in drafting the manuscript. Both authors read and approved the final manuscript.

\section{Acknowledgements}

This research was supported by the MSIT(Ministry of Science and ICT), Korea, under the ITRC(Information Technology Research Center) support program(IITP-2018-2016-0-00311) supervised by the IITP(Institute for Information \& communications Technology Promotion). 
Competing interests

The authors declare that they have no competing interests.

Availability of data and materials

Not applicable.

Ethics approval and consent to participate

Not applicable.

Funding

Not applicable.

\section{Publisher's Note}

Springer Nature remains neutral with regard to jurisdictional claims in published maps and institutional affiliations.

Received: 30 January 2018 Accepted: 6 April 2018

Published online: 26 April 2018

\section{References}

1. Gubbi J et al (2013) Internet of things (IOT): a vision, architectural elements, and future directions. Future Gener Comput Syst 29(7):1645-1660

2. Moon SY, Park JH (2016) Efficient hardware-based code convertor of a quantum computer. J Converg 7:1-9

3. Huh JH (2017) PLC-based design of monitoring system for ICT-integrated vertical fish farm. Human-centric Comput Inf Sci 7(1):1-19

4. Wu M et al (2010) Research on the architecture of Internet of things. In: 3rd international conference on advanced computer theory and engineering (ICACTE), vol 5. 2010. New York, IEEE

5. Koshizuka N, Sakamura K (2010) Ubiquitous ID: standards for ubiquitous computing and the internet of things. IEEE Pervasive Comput 9(4):98-101

6. Huh J-H, Seo K (2017) An indoor location-based control system using bluetooth beacons for loT systems. Sensors 17(12):2917

7. Gellersen HW, Schmidt A, Beigl M (2002) Multi-sensor context-awareness in mobile devices and smart artifacts. Mob Netw Appl 7(5):341-351

8. Healey J, Picard R (2000) SmartCar: detecting driver stress. In: 2000 proceedings of the 15 th international conference on pattern recognition, vol 4. IEEE

9. Abowd GD et al (1999) Towards a better understanding of context and context-awareness. In: International symposium on handheld and ubiquitous computing. Springer, Berlin, Heidelberg

10. Gerla M et al (2014) Internet of vehicles: from intelligent grid to autonomous cars and vehicular clouds. In: 2014 IEEE world forum on internet of things (WF-IOT). IEEE

11. Kim S, Hwang KI (2017) Design of real-time CAN framework based on plug and play functionality. J Inf Process Syst 13(2):348-359

12. Lien C et al (2009) Home appliance energy monitoring and controlling based on power line communication. In: Digest of technical papers international conference on consumer electronics, ICCE'09, Las Vegas, 10-14 January 2009. IEEE

13. Jie Y et al (2013) Smart home system based on iot technologies. In: 2013 fifth international conference on computational and information sciences (ICCIS), Shiyang, 21-23 June 2013. IEEE

14. Kidd C et al (1999) The aware home: a living laboratory for ubiquitous computing research. In: international workshop on cooperative buildings. Integrating information, organizations, and architecture, pp 191-198

15. Debes C, Merentitis A, Sukhanov S, Niessen M et al (2016) Monitoring activities of daily living in smart homes understanding human behavior. IEEE Signal Process Mag 33(2):81-94

16. Hussain M, Afzal M, Khan WA, Lee S et al (2012) Clinical decision support service for elderly people in smart home environment. In: 2012 12th international conference on control, automation, robotics and vision (ICARCV). pp 678-683

17. Jalal A, Sarif N, Kim JT, Kim TS (2013) Human activity recognition via recognized bodyparts of human depth silhouettes for residents monitoring services at smart home. Indoor Built Environ 22(1):271-279

18. Mocanu I, Florea AM (2011) A multi-agent system for human activity recognition in smart environments. In: Brazier FMT, Nieuwenhuis K, Pavlin G, Warnier M, Badica C (eds) Intelligent distributed computing, vol 382. Springer, Berlin, pp 291-301

19. Libal V, Ramabhadran B, Mana N, Pianesi F et al (2009) Multimodal classifcation of activities of daily living inside smart homes. In: Omatu S, Rocha MP, Bravo J, Fernandez F, Corchado E, Bustillo A, Corchado JM (eds) Distributed computing, artifcial intelligence, bioinformatics, soft computing, and ambient assisted living, pt II, proceedings, vol 5518. Springer, Berlin, p 687

20. Ni Q, Garcia Hernando AB, de la Cruz IP (2015) The Elderly's independent living in smart homes: a characterization of activities and sensing infrastructure survey to facilitate services development. Sensors 15(5):11312-11362

21. Dersingh A (2014) IEEE human movement detection using home network information and events on smartphones. In: 2014 international conference on electronics, information and communications (ICEIC), p 2

22. Noury $\mathrm{N}$ et al (2000) Monitoring behavior in home using a smart fall sensor and position sensors. In: 1 st annual international, conference on microtechnologies in medicine and biology, Lyon, 12-14 October 2000. IEEE 
23. Chahuara P, Fleury A, Portet F, Vacher M (2016) On-line human activity recognition from audio and home automation sensors: comparison of sequential and non-sequential models in realistic smart homes. J Ambient Intell Smart Environ 8:399-422

24. Biswas MAR, Robinson MD, Fumo N (2016) Prediction of residential building energy consumption: a neural network approach. Energy 117:84-92

25. Mba L, Meukam P, Kemajou A (2016) Application of artifcial neural network for predicting hourly indoor air temperature and relative humidity in modern building in humid region. Energy Build 121:32-42

26. Zhong BC, Lu K, LV DH, Luo J (2015) Short-term prediction of building energy consumption based on GALM neural network. In: Liu M, Zhang X (eds) Proceedings of the international conference on advances in mechanical engineering and industrial informatics, vol 15. Atlantis Press, Paris, pp 867-871

27. Yuce B, LI HJ, Rezgui Y, Petri I et al (2014) Utilizing artifcial neural network to predict energy consumption and thermal comfort level: an indoor swimming pool case study. Energy Build 80:45-56

28. Lv DH, Zhong BC, Luo J (2015) Application of GLBP algorithm in the prediction of building energy consumption. Int J Adv Comput Sci Appl 6(6):45-48

29. Hsu CL, Chen KY (2009) Practical design of intelligent remote-controller with speech-recognition and self-learning function. In: IEEE 2009 international conference on machine learning and cybernetics. pp 3361-3368. https://doi. org/10.1109/icmlc.2009.5212758. ISBN 978-1-4244-3702-3

30. Goldberg David E, Holland John H (1988) Genetic algorithms and machine learning. Mach Learn 3(2):95-99

31. Yun J et al (2015) A device software platform for consumer electronics based on the internet of things. IEEE Trans Consum Electron 61(4):564-571

32. Telecom ITU (1996) Standardization sector of ITU, video coding for low bitrate communication. Draft ITU-T recommendation $\mathrm{H} 263$

33. Want R (2006) An introduction to RFID technology. IEEE Pervasive Comput 5(1):25-33

34. Midilli A, Dincer I, Ay Murat (2006) Green energy strategies for sustainable development. Energy Policy 34(18):3623-3633

35. Huh JH (2017) Smart grid test bed using OPNET and power line communication," advances in computer and electrical engineering. IGI Global, Pennsylvania, pp 1-425

36. Huh JH, Otgonchimeg S, Seo K (2016) Advanced metering infrastructure design and test bed experiment using intelligent agents: focusing on the PLC network base technology for smart grid system. J Supercomput 72(5):1862-1877

37. Abadi M et al (2016) TensorFlow: a system for large-scale machine learning. In: 12th USENIX symposium on operating systems design and implementation, Savannah, 2-4 November 2016, pp 265-283

38. Abadi M et al (2016) Tensorflow: large-scale machine learning on heterogeneous distributed systems. arXiv preprint arXiv:1603.04467

39. OCEAN (open alliance for iot standard). http://www.iotocean.org/. Accessed 25 July 2017

40. wideeyed NEVER blog. Learning from celsius and fahrenheit temperature measurement data. https://blog.naver. com/wideeyed/. Accessed 18 Nov 2017

41. Jupyter http://jupyter.org/. Accessed 28 Jan 2018

\section{Submit your manuscript to a SpringerOpen ${ }^{\circ}$ journal and benefit from:}

- Convenient online submission

- Rigorous peer review

- Open access: articles freely available online

- High visibility within the field

- Retaining the copyright to your article

Submit your next manuscript at $\boldsymbol{\Delta}$ springeropen.com 